\title{
Short communication: The effects of supplementation of various n-3 fatty acids to late-pregnant dairy cows on plasma fatty acid composition of the newborn calves
}

\author{
U. Moallem ${ }^{\star 1}$ and M. Zachut ${ }^{\star}+$ \\ *Department of Ruminant Science, Institute of Animal Sciences, Volcani Center, Bet Dagan 50250, Israel \\ †Department of Animal Science, the Robert H. Smith Faculty of Agriculture, Food and Environmental Quality Sciences, \\ the Hebrew University of Jerusalem, Rehovot 76100, Israel
}

\begin{abstract}
The passage of long-chain fatty acids (FA) through the placenta in ruminants is limited. However, essential long-chain polyunsaturated FA, and especially n-3 FA, are crucial for normal development of the bovine fetus; therefore, uptake of these FA by the embryo must occur during pregnancy. The objectives of the present study were to examine the effects of enrichment of dam plasma with various n-3 FA during late gestation on newborn calf plasma FA composition. Twenty-seven multiparous cows at $256 \mathrm{~d}$ of pregnancy were divided into 3 groups and fed encapsulated fats as follows: 1) control: supplemented at $240 \mathrm{~g} / \mathrm{d}$ per cow with saturated FA; 2) flaxseed oil (FLX): supplemented at 300 $\mathrm{g} / \mathrm{d}$ per cow with fat that provided $56.1 \mathrm{~g} / \mathrm{d}$ per cow of $\alpha$-linolenic acid (ALA) from flaxseed oil; and 3) fish oil (FO): supplemented at $300 \mathrm{~g} / \mathrm{d}$ per cow with fat that provided 5.8 and $4.3 \mathrm{~g} / \mathrm{d}$ per cow eicosapentaenoic acid (EPA) and docosahexaenoic acid (DHA) from fish oil, respectively. Blood samples were taken from dams twice weekly and from calves immediately after calving before first colostrum. The FA composition in plasma was determined in dams at the last sample before parturition, on average $2 \mathrm{~d}$ before calving. Feeding cows with FLX resulted in a 2.6-fold increase in the proportion of ALA in dam plasma as compared with the control. The proportion of EPA in cow plasma was not different between groups; however, the percentage of docosapentaenoic acid (DPA) was 1.4 and 2 times higher, respectively, in cows fed FLX and FO than in the controls. In addition, the plasma proportion of DHA was 15 times higher in FO cows than in controls. In calves, no differences between groups were observed in the plasma proportions of ALA and EPA; however, the proportion of DHA was 1.9 times higher in the
\end{abstract}

Received February 22, 2012.

Accepted March 11, 2012

${ }^{1}$ Corresponding author: uzim@volcani.agri.gov.il
FO calves than in controls. Across treatments, data showed no correlation between the proportions of ALA, EPA, and DPA in dam and calf plasma; however, positive correlation was demonstrated between dams and calves in DHA proportion $(\mathrm{r}=0.55)$. In conclusion, the distinct plasma FA profile in newborn calves compared with dams was apparently due to low permeability of the bovine placenta to polyunsaturated FA. Enriching late-gestation dairy cows with n-3 FA increased the proportion of DHA, but not ALA, in newborn calf plasma, probably because of the essentiality of DHA to fetal development.

Key words: n-3, fetus essential fatty acid

\section{Short Communication}

In general, epitheliochorial placentas are less permeable to FFA than hemochorial ones (Battaglia and Meschia, 1988). Moreover, the nutrient requirements for fat deposition in bovine fetuses are relatively insignificant (Bell, 1995); therefore, placental transport of short- and long-chain FA is limited (Bell, 1993). In sheep, the modest rate of fetal fat deposition has been attributed to placental impermeability to long-chain FA preformed in the maternal circulation (Elphick et al., 1979), and to a greatly reduced capacity for de novo FA synthesis in fetal adipose tissue during late pregnancy (Vernon et al., 1981). However, long-chain PUFA with 20- and 22-carbon atoms play an important physiological role during pregnancy, both for the pregnant dam and for the unborn calf, as they are critical for the development of the central nervous system (Koletzko et al., 2007) and the reproductive system (Stoffel et al., 2008). Although the passage of PUFA through the bovine placenta is minimal in ruminants, the FA of the maternal circulation are the main significant source of FA to the fetus (Campbell et al., 1994). Limited information is available regarding transfer of specific PUFA from dam to fetus in cattle; therefore, the objectives of the present work were to examine 1) the plasma FA composition in newborn calves in comparison to dams 
and 2) the effects of supplementation of various n-3 FA $[\alpha$-linolenic acid (ALA), eicosapentaenoic acid (EPA), and docosahexaenoic acid (DHA)] to late-pregnant cows on FA composition in plasma of the newborn calves.

The Volcani Center Animal Care Committee approved all the procedures involving animals. Twentyseven dry multiparous Israeli-Holstein cows at the Volcani Center experimental farm (Bet Dagan, Israel), $249 \mathrm{~d}$ pregnant, were group housed in shaded outdoor pens with adjacent outside yards, which were equipped with a real-time electronic individual feeding system. At $256 \mathrm{~d}$ of pregnancy, following $7 \mathrm{~d}$ of adaptation to the individual feeding system, the cows were divided into 3 treatment groups and were stratified randomly within stratum. Strata were defined according to milk yield and fat percentage during the first 100 DIM of the previous lactation, parity, and BW at dry-off. The dietary treatments, which continued from $256 \mathrm{~d}$ of pregnancy to calving, were 1) control: supplemented at $240 \mathrm{~g} / \mathrm{d}$ per cow with encapsulated fat containing saturated FA; 2) flaxseed oil (FLX): supplemented at $300 \mathrm{~g} / \mathrm{d}$ per cow with encapsulated fat that provided ALA from flaxseed oil at $56.1 \mathrm{~g} / \mathrm{d}$ per cow; and 3) fish oil (FO): supplemented at $300 \mathrm{~g} / \mathrm{d}$ per cow with encapsulated fat that provided EPA and DHA from fish oil at 5.8 and $4.3 \mathrm{~g} / \mathrm{d}$ per cow, respectively. Diets were isonitrogenous and isoenergetic, and etherextracted contents were also similar. The diet provided $1.45 \mathrm{Mcal} / \mathrm{kg}$ of DM, $13 \% \mathrm{CP}, 54.4 \%$ crude NDF, and $3.15 \%$ ether extracts. Fatty acid compositions of the fat supplements are presented in Table 1. Cows were individually fed a TMR daily at $1100 \mathrm{~h}$. The fat supplements were individually hand mixed into the TMR, and the amounts of feed offered and refused were recorded daily. Blood samples were collected from cows twice weekly until calving and from calves immediately after calving, before colostrum offering. All blood samples were collected from the jugular vein into vacuum tubes containing lithium heparin (Becton Dickinson Systems, Cowley, UK). Plasma was collected after centrifugation at $1,500 \times g$ for $20 \mathrm{~min}$, and stored at $-32^{\circ} \mathrm{C}$ pending analysis. The FA composition in plasma of dams was determined in the last samples before parturition, and gas chromatography was performed as described previously (Zachut et al., 2010). The FA composition of the control group formed the basis for general comparison between dam and calf FA profiles.

The plasma FA compositions in dams and calves were analyzed with the GLM procedure of SAS Institute (2002). The model used was

$$
\mathrm{Y}_{\mathrm{ijk}}=\mu+\mathrm{T}_{\mathrm{i}}+\mathrm{C}_{\mathrm{j}}+\mathrm{C}(\mathrm{T})_{\mathrm{ij}}+\mathrm{E}_{\mathrm{ijk}}
$$

where $\mathrm{Y}_{\mathrm{ijk}}$ is the dependent variable, $\mu=$ overall mean, $\mathrm{T}_{\mathrm{i}}=$ treatment effect, ${ }_{\mathrm{i}}=1$ to $3, \mathrm{C}_{\mathrm{j}}=$ cow effect, $\mathrm{C}(\mathrm{T})_{\mathrm{ij}}=\operatorname{cow}_{\mathrm{j}}$ nested in treatment ${ }_{\mathrm{i}}$, and $\mathrm{E}_{\mathrm{ijk}}=$ random residual. Mean separation was performed by using the PDIFF procedure with a Tukey adjustment in SAS (version 9.2). The PROC REG procedure of SAS Institute (2002) was used for correlation analysis. Least squares means and adjusted standard error of the means are presented in Table 2 and $P<0.05$ was accepted as significant.

The fat supplementation period averaged $22 \mathrm{~d}(\mathrm{SD}=$ 4). The DMI during this period was $12.7,12.3$, and 11.9 $\mathrm{kg} / \mathrm{d}(\mathrm{SEM}=0.37, P<0.3)$ for control, FLX, and FO, respectively. The dietary fat supplementations altered the plasma FA profile in the pregnant cows (Table 2). Feeding cows with FLX resulted in a 2.6-fold increase in the proportion of ALA in plasma as compared with the control. The proportion of EPA in cow plasma was not different between groups; however, the percentage of docosapentaenoic acid (DPA) was 1.4 and 2 times higher, respectively, in cows fed FLX and FO than in the controls $(P<0.001)$. In addition, the plasma proportion of DHA was 15 times higher in FO cows than in controls $(P<0.001)$.

Comparison between the plasma FA compositions of dams and calves fed the control diet revealed that the proportions of SFA and MUFA in plasma of the control cows were lower than in control calves $(43.2$ vs. $50.0 \%$ and 16.2 vs. $30.6 \%$, respectively; $\mathrm{SEM}=2.0, P<0.02)$, whereas the proportion of PUFA was twice as high in dams as in calves (40.6 vs. $19.3 \%$, respectively; SEM $=0.85, P<0.001)$. The proportions of total $\mathrm{n}-3$ and total n-6 PUFA were higher in dams than in calves: 2.3 vs. $1.1 \%$ and 36.6 vs. $13.3 \%$, respectively $(P<0.001)$.

Table 1. Fatty acid composition (g/100 g of FA) of the fat supplements

\begin{tabular}{lccr}
\hline FA & Control $^{1}$ & FLX $^{2}$ & FO $^{3}$ \\
\hline C14:0 & 1.0 & 0.1 & 2.2 \\
C15:0 & - & - & 0.1 \\
C16:0 & 64.2 & 9.2 & 15.5 \\
C16:1 & - & - & 2.5 \\
C16:2 & - & - & 0.2 \\
C17:0 & 34.8 & 53.0 & 0.3 \\
C18:0 & - & 8.1 & 53.3 \\
C18:1 & - & 6.1 & 12.7 \\
C18:2n-6 & - & 23.4 & 3.2 \\
C18:3n-3 & - & 0.1 & 3.3 \\
C20:0 & - & - & 0.3 \\
C20:3 & - & - & 0.6 \\
C20:5n-3 & - & - & 2.5 \\
C22:1 & - & - & 1.5 \\
C22:6n-3 & & & 1.9 \\
\hline
\end{tabular}

${ }^{1}$ Encapsulated fat containing SFA.

${ }^{2}$ Encapsulated fat containing flaxseed oil.

${ }^{3}$ Encapsulated fat containing fish oil. 
Table 2. Fatty acid composition ( $\mathrm{g} / 100 \mathrm{~g}$ of $\mathrm{FA}$ ) of maternal plasma before calving and newborn calves before first colostrum $^{1}$

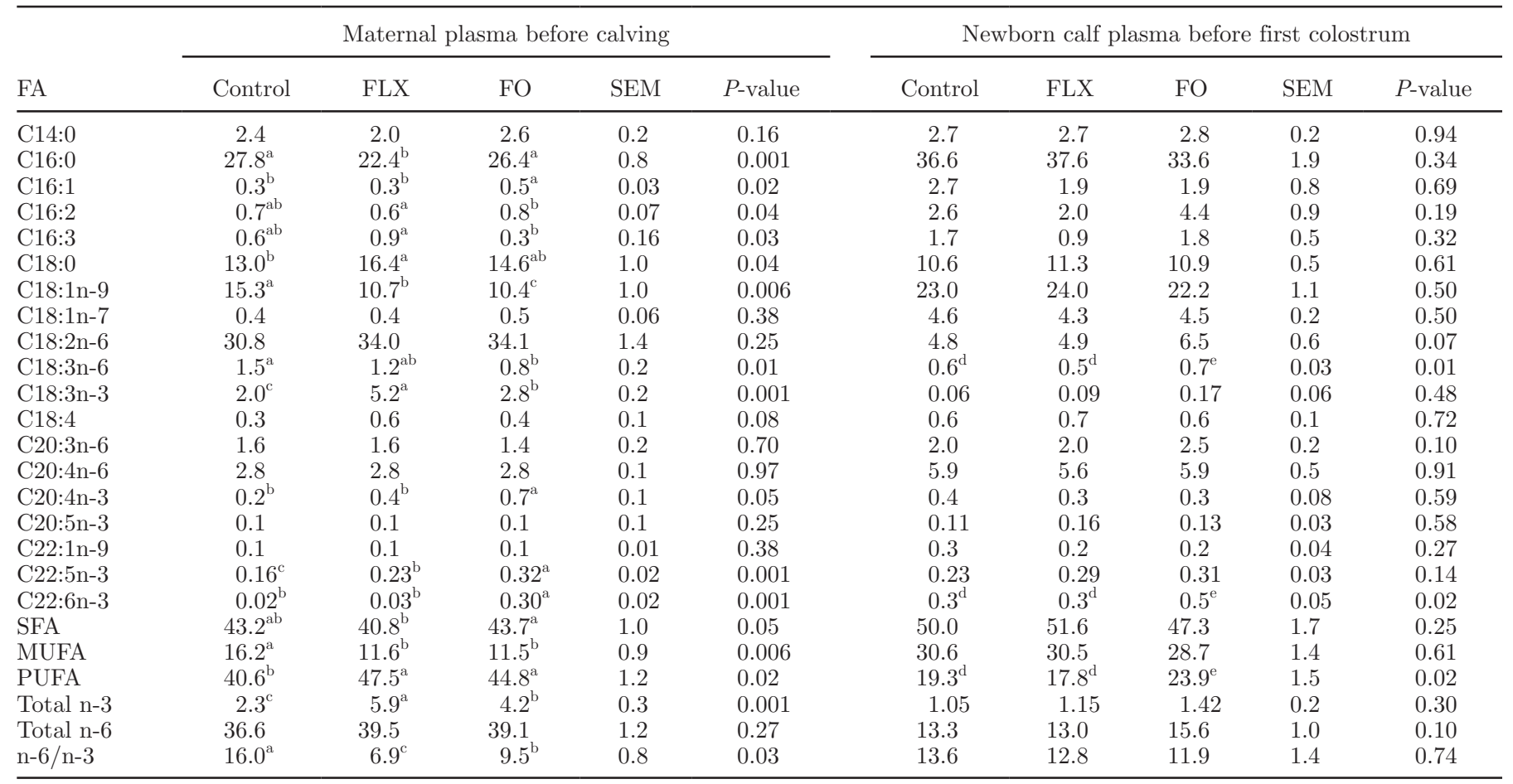

${ }^{\mathrm{a}-\mathrm{c}}$ Within rows for maternal plasma, means with different letter superscripts are statistically different $(P<0.05)$.

${ }^{\mathrm{d}, \mathrm{e}}$ Within rows for calf plasma, means with different letter superscripts are statistically different $(P<0.05)$.

${ }^{1}$ Control = cows supplemented with encapsulated fat containing SFA during late pregnancy; FLX = cows supplemented with encapsulated fat containing flaxseed oil during late pregnancy; FO = cows supplemented with encapsulated fat containing fish oil during late pregnancy.

As shown in Table 2, the proportion of DHA was 1.9 times higher in the FO calves than in controls, but no differences between groups were observed in the plasma proportions of ALA, EPA, and DPA. Across-treatments data showed no correlation between the proportions of ALA, EPA, and DPA in dam and calf plasma; however, positive correlation was demonstrated between dams and calves in DHA proportion in plasma $(\mathrm{r}=0.55 ; P<$ $0.03)$. It was also found that the total PUFA contents were 6.1 and $4.6 \%$ higher in the FO calves than in the FLX and control calves, respectively $(P<0.02)$.

In this study, we found several differences between dams and calves in the proportions of FA in their plasma: the proportion of PUFA in cow plasma was twice that in calf plasma, and total n- 3 and n- 6 FA concentrations were higher in dams than in calves. Also, in sheep, the plasma FA composition of the fetal lamb is very different than that of the ewe; the proportion of linolenic acid $(\mathbf{L n A})$ is markedly decreased and the proportion of arachidonic acid (ARA) is much higher in the fetal lamb (Shand and Noble, 1981). The proportion of DPA in dam plasma was higher both in FLX and in FO cows as compared with controls, which implies elongation of ALA and EPA, respectively, that were provided in the dietary supplement by the cows' livers.
Nevertheless, the percentage of DPA in calf plasma was not significantly elevated in the FLX and FO groups as compared with the controls. Interestingly, the ALA was barely transferred from the dam into calf blood (Table 2 ). Moreover, the percentage of DHA, which can be synthesized from ALA by elongation and desaturation, in plasma of the FLX calves was not elevated. This is consistent with the findings of de Groot et al. (2004), that supplementation of pregnant women with ALA did not result in enhanced levels of DHA in umbilical cord blood. We also observed a higher proportion of DHA in plasma of control calves than in that of dams, and the plasma proportion of DHA was even higher, at $0.5 \%$ of total FA, in the FO calves than in the control calves (Table 2). In addition, a positive correlation was demonstrated between dams and calves in DHA but not in ALA proportions. The maternal-fetal transfer of FA is considered to depend, in part, on the concentration gradients across the placenta (Hay, 1994), which was also demonstrated in the present study, in which the magnitude of transfer of DHA was influenced by the concentration of this FA in dam blood. Similarly, supplementation of pregnant women with DHA was shown to increase DHA levels in plasma and red blood cell lipids of the infant at birth (Connor et al., 1996). 
The vast differences in FA profiles between dams and calves observed in our study may be explained by 1) placental desaturation of FA or 2) selective uptake of FA by placental FA-binding proteins. In a previous study, placental cotyledons were collected postmortem from ewes at $150 \mathrm{~d}$ of gestation, and it was found that the conversion of $\mathrm{LnA}$ to ARA via $\Delta^{6}$-desaturase was extensive near term (Shand and Noble, 1981); the authors concluded that ovine placental tissue possesses an active desaturase. However, the high activity of placental $\Delta^{6}$-desaturase near parturition can be related to prostaglandin synthesis, as ARA is a precursor of prostaglandins that promote labor, and may not be confined to fetal requirements at that time (Shand and Noble, 1981, 1983). Furthermore, the placental conversion of LnA into ARA does not necessarily indicate transfer of this FA into fetal tissues. The mechanism of extensive placental FA desaturation suggested in the in vitro studies (Shand and Noble, 1981, 1983) is not supported by our findings, as we did not observe higher DHA proportions in plasma of FLX calves (although dams had much higher proportion of plasma ALA, a precursor of DHA) as compared with the control calves. These discrepancies regarding the ability of the ruminant placenta to metabolize long-chain FA should be clarified in further studies. Alternatively, the unique plasma FA composition observed in newborn calves in the present study, and specifically the high plasma proportions of DHA in calves, could be explained by active placental FA-binding proteins. It was demonstrated in humans that placental transfer of DHA involves a multistep process of uptake and intercellular translocation by several membrane-associated and cytosolic FA-binding proteins that prefer n- 6 and n-3 FA over nonessential FA (Campbell et al., 1998; Innis, 2005; Hanebutt et al., 2008). A placental FA-binding protein was also identified in sheep (Campbell et al., 1994); however, to the best of our knowledge, these proteins were not yet identified in the bovine placenta. It is plausible to assume that a similar mechanism enables active transfer of DHA from dam to calf during pregnancy. The beneficial effects of n-3 FA to growing calves were recently demonstrated; feeding calves with milk replacer enriched with ALA resulted in improved growth rates and feed efficiency and altered immune and inflammatory response as compared with control calves (Hill et al., 2011). In the present study, supplementing late-pregnant cows with DHA enriched the newborn plasma with this essential FA, which may exert positive effects on calf health and performance during very early age. In conclusion, dietary DHA, but not ALA, supplemented to the dam diet during the last $3 \mathrm{wk}$ of gestation, influenced the proportion of this
FA in calf blood. Enriching the newborn with this essential FA may exert positive effects on calf health and performance during very early age. However, further sophisticated studies are required to investigate the exact mechanism of transfer of PUFA, and specifically of DHA, through the bovine placenta and the effects on calf health and performance.

\section{REFERENCES}

Battaglia, F. C., and G. Meschia. 1988. Fetal nutrition. Annu. Rev. Nutr. 8:43-61.

Bell, A. W. 1993. Pregnancy and fetal metabolism. Page 405 in Quantitative Aspects of Ruminant Digestion and Metabolism. J. M. Forbes and J. France, ed. CAB International, Oxford, UK.

Bell, A. W. 1995. Regulation of organic nutrient metabolism during transition from late pregnancy to early lactation. J. Anim. Sci. 73:2804-2819.

Campbell, F. M., P. G. Bush, J. H. Veerkamp, and A. K. Dutta-Roy. 1998. Detection and cellular localization of plasma membraneassociated and cytoplasmic fatty acid-binding proteins in human placenta. Placenta 19:409-415.

Campbell, F. M., M. J. Gordon, and A. K. Dutta-Roy. 1994. Plasma membrane fatty acid-binding protein $\left(\mathrm{FABP}_{\mathrm{pm}}\right)$ of the sheep placenta. Biochim. Biophys. Acta 1214:187-192.

Connor, W. E., R. Lowensohn, and L. Hatcher. 1996. Increased docosahexaenoic acid levels in newborn infants by administration of sardines and fish oil during pregnancy. Lipids 31:S183-S187.

de Groot, R. H., G. Hornstra, A. C. van Houwelingen, and F. Roumen. 2004. Effect of alpha-linolenic acid supplementation during pregnancy on maternal and neonatal polyunsaturated fatty acid status and pregnancy outcome. Am. J. Clin. Nutr. 79:251-260.

Elphick, M. C., D. Hull, and F. Broughton Pipkin. 1979. The transfer of fatty acids across the sheep placenta. J. Dev. Physiol. 1:31-45.

Hanebutt, F. L., H. Demmelmair, B. Schiessl, E. Larqué, and B. Koletzko. 2008. Long-chain polyunsaturated fatty acid (LC-PUFA) transfer across the placenta. Clin. Nutr. 27:685-693.

Hay, W. W. Jr. 1994. Placental transport of nutrients to the fetus. Horm. Res. 42:215-222

Hill, T. M., M. J. VandeHaar, L. M. Sordillo, D. R. Catherman, H. G. Bateman, and R. L. Schlotterbeck. 2011. Fatty acid intake alters growth and immunity in milk-fed calves. J. Dairy Sci. 94:39363948 .

Innis, S. M. 2005. Essential fatty acid transfer and fetal development. Placenta 26(Suppl. A):S70-S75.

Koletzko, B., E. Larqué, and H. Demmelmair. 2007. Placental transfer of long-chain polyunsaturated fatty acids (LC-PUFA). J. Perinat. Med. 35(Suppl. 1):S5-S11.

SAS Institute. 2002. SAS User's Guide. Version 9.2. SAS Institute Inc., Cary, NC.

Shand, J. H., and R. C. Noble. 1981. The metabolism of 18:0 and $18: 2(\mathrm{n}-6)$ by the ovine placenta at 120 and 150 days of gestation. Lipids 16:68-71.

Shand, J. H., and R. C. Noble. 1983. The characterization of the linoleic acid desaturation and elongation system in ovine placental tissue. Int. J. Biochem. 15:1367-1371.

Stoffel, W., B. Holz, B. Jenke, E. Binczek, R. H. Günter, C. Kiss, I. Karakesisoglou, M. Thevis, A. A. Weber, S. Arnhold, and K. Addicks. 2008. $\Delta^{6}$-Desaturase (FADS2) deficiency unveils the role of $\omega 3-$ and $\omega 6$-polyunsaturated fatty acids. EMBO J. 27:2281-2292.

Vernon, R. G., J. P. Robertson, R. A. Clegg, and D. J. Flint. 1981. Aspects of adipose-tissue metabolism in foetal lambs. Biochem. J. 196:819-824.

Zachut, M., A. Arieli, H. Lehrer, L. Livshitz, S. Yakoby, and U. Moallem. 2010. Effects of increased supplementation of n-3 fatty acids to transition dairy cows on performance and fatty acid profile in plasma, adipose tissue, and milk fat. J. Dairy Sci. 93:5877-5889. 